\title{
CARACTERIZAÇÃO DE FILMES POLIMÉRICOS COM ADIÇÃO DE POLPA DE PITAYA VERMELHA (Hylocerus Polyrhizus)
}

\section{CHARACTERIZATION OF POLYMERIC FILMS WITH ADDITION OF RED PITAYA PULP (Hylocerus Polyrhizus)}

\section{J. K. ABREU ${ }^{1}$ e A. B. REIS ${ }^{1 *}$}

${ }^{1}$ UFVJM-Universidade Federal dos Vales do Jequitinhonha e Mucuri, Engenharia Química, Diamantina-MG, Brasil

*Autor correspondente. Universidade Federal dos Vales do Jequitinhonha e Mucuri, Departamento de Engenharia Química, Diamantina, Minas Gerais, Brasil, e-mail: arlete.reis@ict.ufvjm.edu.br(A.B.Reis)

\begin{tabular}{l} 
A R T I C L E I N F O \\
\hline Article history: \\
Received 2020-06-29 \\
Accepted 2020-10-20 \\
Available online 2020-10-20 \\
pa lavras - chave \\
Biopolímeros \\
Quitosana \\
Betalaína \\
\\
ke ywords \\
Biopolymer \\
Chitosan \\
Betalain
\end{tabular}

Betalain

\begin{abstract}
A B S T R A C T
Pitaya is a fruit of epiphyte cacti of the genus Hylocereus, and can be found more easily in Brazilian territory in two different species: White Pitaya (Hylocereus undatus) red bark and white pulp and Red Pitaya (Hylocerus Polyrhizus) and red pulp. The objective of the work was to make polymeric films with the addition of red pitaya pulp in order to detect possible properties of the pulp, that when inserted in the films, give it relevant characteristics for future active packaging. Three different formulations were made: the first formulation containing only $2 \%$ chitosan (by weight); the second formulation containing $2 \%$ chitosan plus $1 \mathrm{~mL}$ of pulp; the third formulation containing $2 \%$ chitosan, plus $5 \mathrm{~mL}$ of pulp. In order to characterize the three film formulations, the following analyzes were performed: Thickness, Water Vapor Permeability (PVA), moisture content and water solubility. At the end, it was observed that the addition of different concentrations of pulp changed the characteristics of biopolymer films.
\end{abstract}

R E S U M O

A Pitaya é um fruto de cactos epífitos do gênero Hylocereus, podendo ser encontradas mais facilmente em território brasileiro em duas diferentes espécies: Pitaya Branca (Hylocereus undatus) casca vermelha e polpa branca e a pitaya vermelha (Hylocerus Polyrhizus) e polpa vermelha. $O$ objetivo do trabalho foi confeccionar filmes poliméricos com adição de polpa de pitaya vermelha afim de detectar possiveis propriedades da polpa que ao serem inseridas nos filmes, dê ao mesmo características relevantes para futuras confecções de embalagens ativas. Foram confeccionadas três formulações distintas: a primeira formulação contendo somente quitosana $2 \%$ (em massa); a segunda formulação contendo quitosana $2 \%$ acrescido de $1 \mathrm{~mL}$ de polpa; a terceira formulação contendo quitosana $2 \%$, acrescida de $5 \mathrm{~mL}$ de polpa. Afim de realizar a caracterização das três formulações de filmes, foram realizadas as seguintes análises: Espessura, Permeabilidade ao Vapor D’água (PVA), teor de umidade e solubilidade em água. Ao final, observou-se que a adição de diferentes concentrações de polpa alterou as características dos filmes biopoliméricos. 


\section{INTRODUÇÃO}

Pitaya é uma fruta exótica natural de cactos epífitos, e por essa característica não necessita de irrigação continua para se desenvolver. Em território brasileiro são conhecidos cerca de 36 gêneros e aproximadamente 230 espécies, e que são cultivadas de várias formas como plantas ornamentais e comestíveis. Entre as espécies comestíveis as espécies que foram utilizadas na elaboração da pesquisa foi a pitaya vermelha (Hylocerus Polyrhizus) caracterizada por ter a casca vermelha e polpa vermelha como mostra a Figura 1.

A pitaya é rica nos pigmentos naturais betalaínas que possuem atividade antioxidante e potencial para serem extraídos e utilizados na indústria alimentícia e cosmética (Adnan; Osman; Hamid, 2011; Esquivel; Stintzing; Carle, 2007; Suh et al., 2014). Possui capacidade antioxidante principalmente pela presença de compostos como o ácido ascórbico, carotenóides e polifenóis, sendo essa ação benéfica bastante visada para combater o estresse oxidativo no corpo, prevenindo doenças degenerativas (Abreu et al., 2012; Constant, 2002).

A betalaína, presente tanto na casca quanto na polpa da pitaya, é um pigmento solúvel em água e contém ácido betalâmico em sua estrutura. Esse pigmento é conhecido pela sua coloração avermelhada e amarelo-alaranjado, assim como pelas propriedades antioxidante, anti-inflamatória e quimiopreventiva. (García-Cruz et al., 2013)

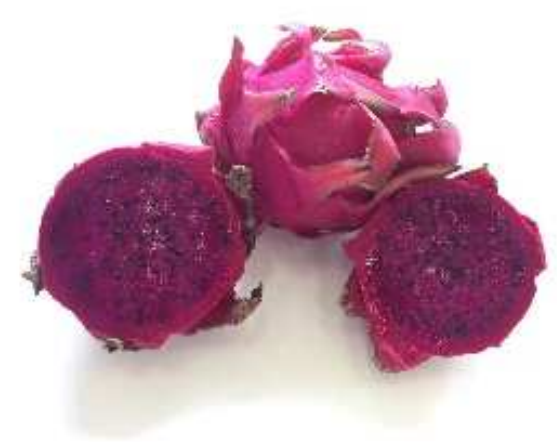

\section{Figura 1 - Pitaya Vermelha (Hylocerus Polyrhizus). (Foto tirada pelo autor, 2019)}

As pitayas se diferem em tamanho, formato e cor da polpa. A polpa da pitaya vermelha, tem um tom avermelhado bem acentuado. A cartela de cores que se obtêm ao analisar as duas espécies de pitaya é devido a presença dos corantes naturais. Portanto, chama-se a atenção para o uso da polpa da pitaya como agente colorante.

Os corantes naturais apresentam numerosas vantagens comparados aos corantes sintéticos, tal como o baixo custo, não toxicidade e sustentabilidade (Shahid, Islam e Mohammad, 2013), representando assim, uma alternativa à possibilidade de substituição de corantes sintéticos, por exemplo.

Outra vertente a ser considerada, visando a obtenção de produtos sustentáveis, seria a substituição dos polímeros sintéticos por biopolímeros, utilizando estes na confecção de filmes biopolíméricos, que possibilite a inserção de aditivos diversos na matriz polimérica, como o biopolímero quitosana.

A utilização de filme biopoliméricos de quitosana, tem despertado grande interesse frente a utilização de polímeros obtidos via síntese química, pois entre outras vantagens, apresenta potencial de estender o tempo de prateleira de produtos perecíveis, como é o caso dos alimentícios (Reis et al., 2005).

A quitosana é um biopolímero, caracterizado por sua não toxidade, biodegradabilidade, biocompatibilidade, além da sua capacidade antifúngica e antimicrobiana. Quimicamente a quitosana é obtida através da desacetilação, via hidrólise básica, da quitina, material presente principalmente no tecido que compõe o exoesqueleto de invertebrados e carapaça de crustáceos (Oliveira e Junior, 2016). Além de ser um polissacarídeo abundante, a quitosana possui boas propriedades para formação de filmes. Sendo hidrofílicos, formam revestimentos com eficiente barreira contra compostos de baixa polaridade, tais como os lipídios; entretanto, não constituem boa barreira a umidade (Kester e Fennema, 1986). De acordo com Laranjeira e Fávere (2009), a hidrofilicidade deste biopolímero está associada à uma grande porcentagem de hidroxilas e radicais amino, presentes em sua estrutura molecular, apresentada na Figura 2. A utilização deste biopolímero em produção de filmes vem sendo estudada por décadas e é justificada por seu baixo custo de produção e versatilidade (Fráguas et al., 2015).

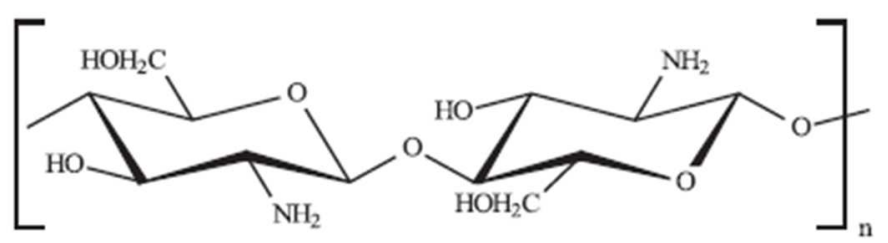

Figura 2 - Estrutura química do biopolimero de quitosana, onde $n$ representa o grau de polimerização. (Assis e Silva, 2003)

Os filmes biopoliméricos a base de quitosana permitem a incorporação de agentes ativos em sua estrutura, abrindo inúmeras possibilidades de se agregar funcionalidade ao filme. Dentre as fontes para obtenção de corantes com funcionalidade, temos as fontes naturais, provindo de matéria orgânica como bagaço de frutas e vegetais, e os corantes sintéticos que vem tendo sua utilização restrita devido aos possíveis efeitos tóxicos que estes podem gerar a longo prazo. Corantes naturais oriundos de frutos, tornam seu uso ainda mais interessante, como é o caso da polpa da pitaya vermelha. A pitaya vermelha está entre as maiores fontes de compostos nitrogenados, que apresentam atividades funcionais, como as betalaínas que são encontrados na polpa da fruta e possuem característica antioxidativas (Drunkler, 2001), portanto podem ser utilizados substituindo antioxidantes quimicamente sintetizados.

Além dessa função de corante que as betalaínas apresentam, elas têm a função de antioxidantes naturais, pois são compostos nitrogenados provenientes do metabolismo secundário. São conhecidas aproximadamente setenta betalaínas, todas com a mesma estrutura fundamental (Figura 3) 1,7 diazoheptamelina. Das setenta conhecidas, cinqüenta são pigmentos vermelhos denominados betacianinas e vinte são pigmentos amarelos, as betaxantinas (Constant et al., 2002). 
<smiles>[R][N+]([R])=CC=C1C=C(C(=O)O)NC(C(=O)O)C1</smiles>

\section{Figura 3 - Estrutura molecular básica das betalaínas (Constant et al., 2002)}

Sob a ótica da inovação tecnológica o emprego de agentes bioativos em embalagens representam uma abordagem inovadora, por meio das embalagens ativas, capazes de garantir a qualidade, integridade e segurança de produtos alimentícios (Yildirim et al., 2018). Segundo Gontard (1997), uma embalagem ativa é aquela que, além de proteger, interage com o produto e, em alguns casos, responde as mudanças que ocorrem no material embalado ou no ambiente ao qual está exposto. Além disso, permitem a liberação ou a absorção de substâncias dentro ou a partir dos alimentos embalados, ou ainda do meio ao redor da embalagem (Vanderroost et al., 2014).

Neste sentido, temos como objetivo o desenvolvimento e caracterização preliminar de filmes a base de quitosana com adição de agentes antioxidantes, realizando a comparação entre o filme padrão de quitosana e o filme produzido com adição da polpa da pitaya vermelha. Para caracterização inicial destes filmes foram realizados alguns ensaios como: análise visual, espessura, solubilidade e permeabilidade em vapor d'água, além de um estudo básico, teórico acerca da atividade antioxidante dos compostos presentes na polpa da pitaya vermelha.

\section{METODOLOGIA}

A seguir são apresentadas as metodologias adotadas neste estudo para o desenvolvimento dos filmes e análises realizadas o a finalidade de determinar a viabilidade de aplicação da polpa da pitaya vermelha na produção de filmes para posterior aplicação em embalagens.

\subsection{Obtenção da Polpa da Pitaya Vermelha}

Utilizou-se a pitaya vermelha adquirida no comércio local. Primeiramente as amostras de pitaya vermelha foram lavadas, separou-se as cascas da polpa e do mesocarpo. Em seguida a polpa passou por uma peneira para que a parte mais liquida fosse separada da parte da mais pastosa que não seria utilizada.

\subsection{Produção dos filmes}

Os produção dos filmes seguiu a técnica de Casting, que consiste na solubilização da quitosana em solução ácida, seguida da evaporação do solvente por secagem em placas de petri. Foram produzidos filmes de $2 \%(\mathrm{~m} / \mathrm{v})$, onde $2 \mathrm{~g}$ de quitosana em pó (Polymar), foram solubilizados em solução de $1,5 \mathrm{~mL}$ de ácido acético (Synth) para $100 \mathrm{~mL}$ de água deionizada, em agitador magnético por 1 hora. A solução formada foi disposta em placas de petri, cada uma contendo $20 \mathrm{~mL}$. As placas de petri foram submetidas a secagem em estufa a $40{ }^{\circ} \mathrm{C}$ por 24 horas. Após a secagem os filmes obtidos foram retirados da placa de petri. Estes filmes foram usados como padrão para as análises realizadas.

Além destas, outras duas formulações foram produzidas, sendo uma com adição de $1 \mathrm{~mL}$ da polpa da pitaya vermelha e outra com adição de $5 \mathrm{~mL}$ da polpa da pitaya vermelha. Na Tabela 1, são apresentadas as nomenclaturas de cada formulação e sua respectiva composição.

\subsection{Análise visual}

A partir da obtenção dos filmes, foi observado característica perceptíveis ao olho humano das amostras. Avaliou-se coloração, fragilidade, e homogeneidade dos filmes. Os filmes que ao serem retirados das placas apresentassem rupturas, não homogeneidades, ou aspecto quebradiço eram descartados.

\subsection{Espessura}

A espessura dos filmes foi medida aleatoriamente em 5 pontos distintos da área do filme, utilizando o micrômetro Electronic Outside Micrometer - Series 3103 0-25mm/0-1", da marca INSIZE. Analisou-se a espessura pela média e pelo desvio padrão dos pontos medidos.

\subsection{Permeabilidade ao vapor d'água (PVA)}

Para permeabilidade ao vapor d'água seguiu-se o método gravimétrico de acordo com a norma ASTM E96-95 (ASTM, 1995), onde o filme foi mantido em dessecador contendo sílica gel por 48 horas, após ser retirado do dessecador o filme foi utilizado para lacrar um recipiente contendo $20 \mathrm{~g}$ de sílica gel, devidamente vedado com auxílio de fita veda rosca, de forma que não ocorresse entrada de umidade, a não ser por permeabilidade do filme.

Os sistemas, foram mantidos em dessecador contendo solução de $\mathrm{NaCl}$ (Synth) com umidade relativa controlada de $75 \%$ e temperatura ambiente. A variação mássica do sistema foi acompanhada durante 5 dias, e o cálculo da permeabilidade foi realizado como mostra a Equação 1 .

$$
P V A=\frac{\Delta m * x}{A * t * \Delta P}
$$

Onde: $\Delta m$ é a variação de massa do sistema; $x$ é a espessura do filme; $A$ é a área do filme em contato com o meio; $t$ é o tempo de exposição; e $\Delta P$ é a variação de pressão.

\subsection{Solubilidade}

A determinação da solubilidade das amostras foram realizadas de acordo com Amaral et al., (2017), onde uma porção de $1 \mathrm{~g}$ de filmes, cortado em pedaços com área igual a $2 \mathrm{~cm}^{2}(1$ $\mathrm{cm} \times 2 \mathrm{~cm}$ ), foi armazenada em dessecador com sílica gel por 7 dias, em seguida inserida em recipiente contendo $80 \mathrm{~mL}$ de água em temperatura ambiente e submetidas a agitação de $200 \mathrm{rpm}$ em manta agitadora por 1 hora. Após agitação a amostra de filme foi seca em estufa a $60^{\circ} \mathrm{C}$, pelo período de 24 horas. A partir das análises do experimento realizado em triplicata, obteve-se a solubilidade das amostras pela Equação 2.

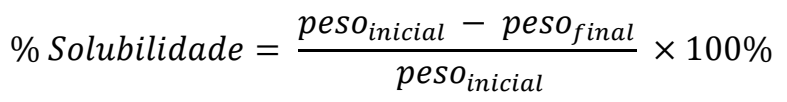


Tabela 1- Composição dos filmes e nomenclatura correspondente.

$\begin{array}{llll}\text { Nomenclatura } & \text { Q2 } & \text { Q2-P1 } & \text { Q2-P5 } \\ \text { Água / mL } & 100,00 & 100,00 & 100,00 \\ \text { Quitosana / g } & 2,00 & 2,00 & 2,00 \\ \text { Ác. acético / mL } & 1,50 & 1,50 & 1,50\end{array}$

\section{RESULTADOS}

Os resultados obtidos no presente estudo para as análises realizadas nas amostras de filmes Q2, Q2-P1, Q2-P5, estão disponíveis a seguir.

\subsection{Análise Visual}

Os filmes Q2, Q2-P1 e Q2-P5 produzidos pelo método de Casting são apresentadas a seguir na Figura 4, as fotografias foram tiradas logo após a retirada dos filmes das placas de petri, após etapa de secagem, com o auxílio de um smartphone.

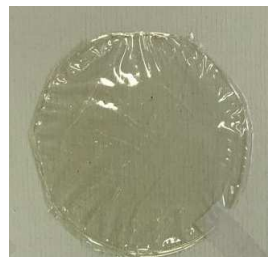

a) Q2

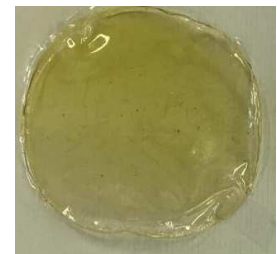

b) Q2-P1

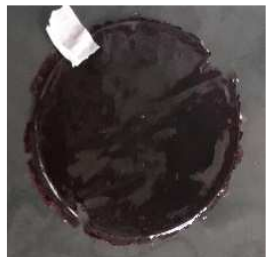

c) Q2-P5
Figura 3- Amostra de filmes produzidos - a) Q2, b) Q2P1, c) Q2-P5.

Por inspeção visual, os filmes apresentaram-se íntegros e homogêneos. Em relação a coloração os filmes Q2 apresentaram maior transparência, porém apesar de apresentar certa transparência possui uma coloração amarelada muito suave, os filmes Q2-P1 apresentaram coloração amarelo intenso. Os filmes Q2-P5 demonstrou característica mais rígida e espessa, e um tom violeta.

\subsection{Espessura}

Os filmes a base de quitosana apresentaram, segundo análise, os valores de espessura média como mostra Tabela 2. A incorporação da polpa, na solução filmogênica, resultou em um aumento da espessura dos filmes em relação ao filme padrão.

Tabela 2- Espessura média das amostras de filmes.

$\begin{array}{cl}\text { Amostra } & \text { Espessura média / mm } \\ \text { Q2 } & 0,045 \pm 0,001^{\mathrm{a}} \\ \text { Q2-P1 } & 0,064 \pm 0,001^{\mathrm{b}} \\ \text { Q2-P5 } & 0,083 \pm 0,001^{\mathrm{c}}\end{array}$

Nota: Resultados que apresentam letras diferentes implicam diferença significativa $(\mathrm{P}<0,05)$ entre as amostras, pelo teste estatístico de Tukey.

\subsection{Permeabilidade ao vapor d'água (PVA)}

A análise de PVA foi realizada em quadruplicada para as amostras de filme Q2, Q2-P1 e Q2-P5 uma área do filme foi $0,0056745 \mathrm{~m}^{2}$ e a variação de pressão de $2,3 \mathrm{kPa}$ ocasionada pela diferença entre as umidades relativas dos meios interno e externo, obteve-se os resultados de PVA apresentados na Tabela 3. Foi possível observar que a permeabilidade é consideravelmente maior nos filmes produzidos com a adição da polpa da pitaya vermelha.

Tabela 3 - Permeabilidade Vapor d'Água.

$\begin{array}{cl}\text { Amostra } & \text { PVA } / \mathrm{g} \cdot \mathrm{mm} \cdot \mathrm{m}^{-2} \cdot \mathrm{h}^{-1} \cdot \mathrm{kPa}^{-1} \\ \text { Q2 } & 0,076 \pm 0,025^{\mathrm{d}} \\ \text { Q2-P1 } & 0,069 \pm 0,010^{\mathrm{c}} \\ \text { Q2-P5 } & 0,060 \pm 0,010^{\mathrm{c}}\end{array}$

Nota: Resultados que apresentam letras diferentes implicam diferença significativa $(\mathrm{P}<0,05)$ entre as amostras, pelo teste estatístico de Tukey.

Comparando-se os filmes Q2, Q2-P1 e Q2-P5 podemos inferir que a adição da polpa da pitaya vermelha influenciou na diminuição da permeabilidade. Para embalagens de alimentos é interessante que a permeabilidade ao vapor d'água seja a menor possível.

\subsection{Solubilidade}

Pode-se observar que as amostras possuem diferentes percentuais de solubilidade em água. Os filmes Q2-P1 e Q2-P5, possuem característica de solubilidade significativamente maior, como mostra a Tabela 4.

Tabela 4 - Solubilidade em água.

\begin{tabular}{cl}
\hline Amostra & Solubilidade / \% \\
Q2 & $11,910 \pm 0,100^{\mathrm{a}}$ \\
Q2-P1 & $23,100 \pm 0,100^{\mathrm{b}}$ \\
Q2-P5 & $70,130 \pm 0,100^{\mathrm{c}}$
\end{tabular}

Nota: Resultados que apresentam letras diferentes implicam diferença significativa $(\mathrm{P}<0,05)$ entre as amostras, pelo teste estatístico de Tukey.

\section{DISCUSSÃ̃}

Pelo aspecto visual os filmes, apresentaram boas características de homogeneidade e integridade. A coloração do filme interfere diretamente na aceitação do produto pelo consumidor, os filmes de polpa de pitaya vermelha possuem coloração amarela intenso atrativa e coloração violeta, além disso pode interferir na incidência de luminosidade no produto. 
A adição da polpa da pitaya alterou de forma significativa a espessura do filme de quitosana, essa característica do filme pode variar dependendo das características do produto. De acordo com Jiménez et al., (2012) é esperado uma espessura mais homogênea a fim de evitar problemas mecânicos que possam comprometer a segurança do produto. De forma geral as amostras testadas apresentaram baixos valores de desvio padrão. A espessura mais fina foi a do filme Q2 com média de $0,045 \mathrm{~mm}$, e a espessura mais alta foi de $0,083 \mathrm{~mm}$ no filme Q2-P5.

Conforme ilustra a Tabela 3, podemos inferir que a permeabilidade ao vapor d'agua do filme diminui em razão do aumento da concentração da polpa da pitaya vermelha. Contudo dada a interação com a polpa da pitaya vermelha o valor médio de PVA não sofre mudança significativa para ambas as concentrações. O maior valor de PVA encontrado foi nos filmes de quitosana e o menor nos filmes de quitosana com adição de $5 \mathrm{~mL}$ de polpa pitaya vermelha.

Com a adição dos compostos da polpa pitaya vermelha não apresentou boa característica de solubilidade, a maior solubilidade observada foi de 70,13\% no filme Q2-P5. Com relação a solubilidade para diferentes concentrações de quitosana pela análise estatística foi possível encontrar diferenças significativas.

\section{CONCLUSÃO}

Com o estudo realizado foi possível desenvolver uma metodologia para obtenção do filme a base do biopolímero quitosana, com adição da polpa da pitaya vermelha. Os resultados obtidos confirmam que a adição da polpa influência significativamente nas propriedades do filme, com potencial de aplicação em embalagens. Análises feitas nas amostras mostram que os filmes possuem espessura homogênea, baixa resistência a permeabilidade ao vapor d'agua. Entretanto, a viabilidade de aplicação está relacionada diretamente às características e necessidades de armazenamento do produto a ser embalado, sendo portanto necessário a realização de estudos mais aprofundados e possíveis ajustes desde a confecção dos filmes até sua aplicação.

\section{AGRADECIMENTOS}

Os autores agradecem ao CNPq, PRPPG/UFVJM e ao GEPAEQ Grupo de Estudos e Pesquisas Aplicadas à Engenharia Química-UFVJM.

\section{REFERÊNCIAS}

ABREU, W. C. et al. Características físico-químicas e atividade antioxidante total de pitaias vermelha e branca. Rev Inst Adolfo Lutz, São Paulo, v. 71, n. 4, p. 656-61, 2012.

ADNAN, L.; OSMAN, A.; HAMID, A. A. Antioxidant activity of different extracts of red pitaia (Hylocereus polyrhizus) seed. Int J Food Prop, New York, v. 14, p. 1171-1181, 2011.

AMARAL, I. B. C.; ARRUDAS, S. R.; DE MEIRA, J. R.; REIS, A. B. Análise do processo difusivo de filmes de quitosana contendo óleo de palmeiras (Aracaceae) do cerrado brasileiro. Revista Unimontes Científica,
Montes Claros, MG, jul./dez. 2017.

AMERICAN SOCIETY FOR TESTING AND MATERIALS-ASTM Book of Standards, Philadelphia, PA. ASTM E96-00 - Standard test methods for determining gas permeability characteristics of plastic film and sheeting, 1995.

AMERICAN SOCIETY FOR TESTING AND MATERIALS-ASTM Book of Standards, Philadelphia, PA. ASTM E96/E 96M-05. Standard Test Methods for water vapor transmission of materials, 2005.

ASSIS, O. B. G.; SILVA, V. L. Caracterização estrutural e da capacidade de absorção de água em filmes finos de quitosana processados em diversas concentrações. Polímeros: Ciência e Tecnologia, v. 13, p. 223-228, 2003.

CONSTANT. P.B.L.; STRINGHETA, P.C.; SANDI, D. Corantes Alimentícios. Boletim Centro de Pesquisa e Processamento de Alimentos., v.20, i2. 1248, 2002.

DRUNKLER, D. A. Estudo da estabilidade de betalaínas em diferentes solventes e em 167 extrato de beterraba (Beta vulgaris L.) adicionado de ciclodextrinas (alfa, ß- e ÿ) e 168 ácidos orgânicos (tânico e gálico). 2001. Dissertação (Mestrado em Ciência dos Alimentos) 200169 Centro de Ciências Agrárias. Programa de Pós-Graduação em Ciência dos Alimentos, 170 Universidade Federal de Santa Catarina, 2001.

ESQUIVEL, P.; STINTZING, F. C.; CARLE, R. Phenolic compound profiles and their corresponding antioxidant capacity of purple pitaya (Hylocereus sp.) genotypes. J Biosci, Bangalore, v. 62, n. 9, p. 636644, 2007.

FRÁGUAS, R. M.; SIMÃO, A. A.; FARIA, P. V.; QUEIROZ, E. DE R.; OLIVEIRA JUNIOR, Ê. N. DE; ABREU, C. M. P. DE. Preparo e caracterização de filmes comestíveis de quitosana. Polímeros. 2015.

GARCIA-CRUZ, L. et al. Physical, chemical and antioxidant activity characterization of Pitaya (Stenocereus pruinosus) Fruits. Plant Foods Hum Nutr, Dordrecht, v. 68, n. 4, p. 403-410, 2013.

GONTARD, N. Active packaging. In: SOBRAL, P.J.A.; CHUZEL, G., eds. Workshop sobre biopolímeros. Pirassununga, FZEA. p. 23-27. 1997.

JIMÉNEZ, A.; FABRA, M. J.; TALENS, P.; CHIRALT, A. Edible and Biodegradable Starch Films: A Review. Food Bioprocess Technology. v.4, p.0-0, 2012.

KESTER, J.J.; FENNEMA, O.R. Edible films and coatings: a review. Food Technology, v. 40, n. 12, p. 47-59, 1986.

LARANJEIRA, M. C. M.; FÁVERE, V. T. Quitosana: Biopolímero funcional com potencial industrial biomédico. Química Nova, Florianópolis, SC, v. 32, p. 672-678, 2009.

LUCHESE, C. L.; SPEROTTO N.; SPADA, J. C.; TESSARO, I. C. Effect of blueberry agroindustrial waste addition to corn starch-based films for the production of a ph-indicator film. International Journal of Biological Macromolecules. 2017.

OLIVEIRA, C.S.; JUNIOR, E.N.O.Produção e caracterização de filmes de quitosana utilizando diferentes ácidos orgânicos. In: XXI Congresso brasileiro de engenharia química, 2016, Fortaleza, CE. Anais [...]. 2016.

REIS, A. B.; YOSHIDA, C. M. P.; FRANCO, T. T. Filmes 
bioativos a partir do polímero quitosana: sistemas de embalagem de papelão. In: $8^{\circ}$ Congresso brasileiro de polímeros, 2005, Águas de Lindóia, SP. Anais [...]. 2005.

SHAHID, M.; ISLAM, S.; MOHAMMAD, F. Recent advancements in natural dye applications: a review. Journal of Cleaner Production, v. 53, p.310-331, 2013.

VANDERROOST, M.; RAGAERT, P; DEVLIEGHERE, F.; MEULENAER, B. Intelligent packaging: The next generation. Trends in Food Science \& Technology, v.39, p. 47-62, 2014.

YILDIRIM, S.; RÖCKER, B.; PETTERSEN, M. K.; NILSEN-NYGAARD, J.; AYHAN, Z.; RUTKAITE, R. Active Packaging Applications for Food. Comprehensive Reviews in Food Science and Food Safety, p. 165-199. 2018. 